\title{
Educational Technology for Collaborative Virtual Environments
}

\author{
Marcio Cunha, Alberto Raposo, Hugo Fuks \\ Department of Informatics, Catholic University of Rio de Janeiro \\ R. M. S. Vicente 225, Gávea - Rio de Janeiro - RJ \\ 22453-900 Brazil \\ marcio@iag.puc-rio.br,abraposo@tecgraf.puc-rio.br,hugo@inf.puc-rio.br
}

\begin{abstract}
$3 D$ information systems for education must be investigated and adopted for a more involving form of learning. It is in this context that we propose the use of Second Life as an environment for collaborative learning and generation of new educational content. Second Life is a simulator of real life and relationships, an entirely three-dimensional virtual world. There, besides interacting with users from the whole world in real time, it is also possible to create one's own objects and businesses, and generate new alternatives of content, exchange of information, and learning.
\end{abstract}

Keywords: CSCW, Collaborative Design, Virtual Worlds.

\section{Introduction}

We live in a world that is increasingly interconnected, and the Internet has developed as an essential vehicle for communication, socialization and creative expression. Virtual worlds such as Second Life [14] represent a possible future for the interaction of human beings [6] in an interconnected global world; students who came of age with the Internet sail naturally in these waters (Figures 1 and 2). These digital natives embrace tools such as Instant Messengers, networked workspaces and multiplayer online games eagerly and naturally. As the students of today will become the leaders of tomorrow, they will of course bring these new technologies to their work environments, making their use an essential part of their businesses' future and routine.

Second Life is a simulator of real life and relationships, an entirely three-dimensional virtual world. There, besides interacting with users from the whole world in real time, it is also possible to create one's own objects and businesses, and even generate new alternatives of content, exchange of information, and learning. In other words, Second Life is what you make of it.

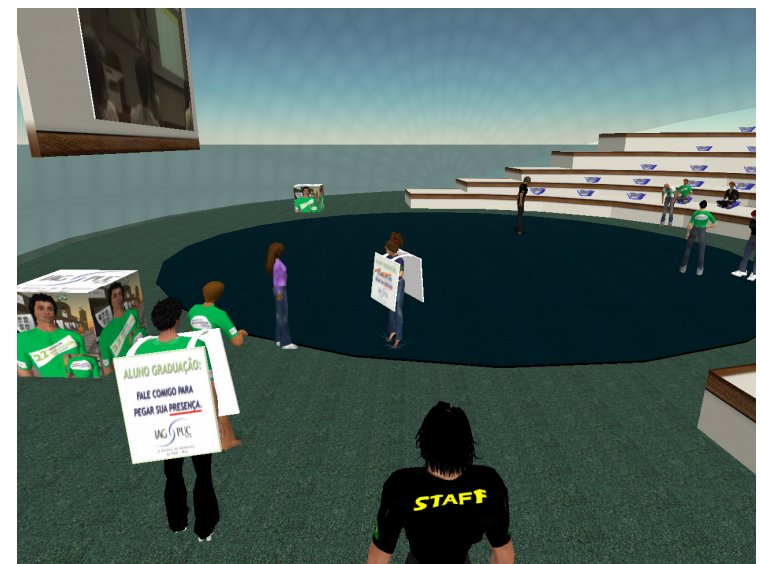

Figure 1 - Students and Staff participating in the Administration Week

Since the opening of Harvard Law School courses in Second Life in 2006 [10], many metaverses offer more and more opportunities for learning and professional updating. Educational institutions and companies invest in the possibilities brought about by these threedimensional tools.

Much of the interest of companies and educational institutions in Second Life or other virtual worlds, besides the possibility of people interaction through their avatars, concerns their video and audio streaming capabilities (Figure 3) [7], which facilitate many activities in distance education. Students can communicate person-to-person by means of instant messages [13] or voice over IP while they listen to an audio or watch a video in the classroom.

From the interest demonstrated by the academic world, one can conclude that Second Life is seen as promising by educational institutions and companies in other fields that intend to expand their brands and businesses into that world. It can be said that distance education has been one of the most viable alternatives regarding the business potential of virtual environments.

978 -1-4244-1651-6/08/\$25.00 C 2008 IEEE 


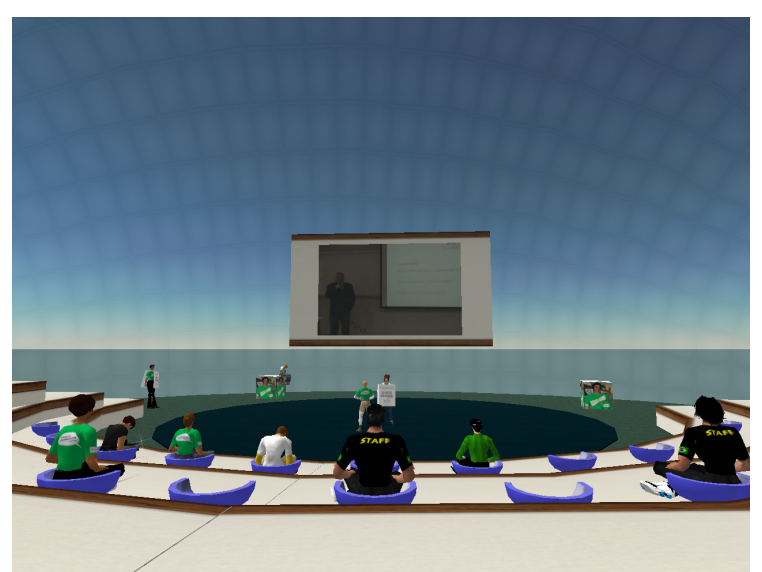

Figure 2 - Students Avatars participating in the Administration Week

\section{Second Life as an Alternative Collaborative Learning Environment}

Traditional collaboration in classrooms, conference rooms and convention centers has being systematically surpassed by collaboration in a global scale. Nowadays encyclopedias, operational systems, mutual funds and even airplanes are being produced by teams made up of thousands of people. While some executives fear the exponential growth of these online communities, this new contribution-based economy demonstrates that such fear is foolish. Visionary companies have exploited collective skills and geniality to stimulate innovation, growth and success.

Through mass collaboration, individuals now create a wide range of free, open code goods and services that anyone can modify or use. Thus they produce new TV programs [4], sequence the human genome, remix their songs, develop software, edit texts and build the imaginable. And this is all developed inside a universe in this case metaverse - called Second Life.

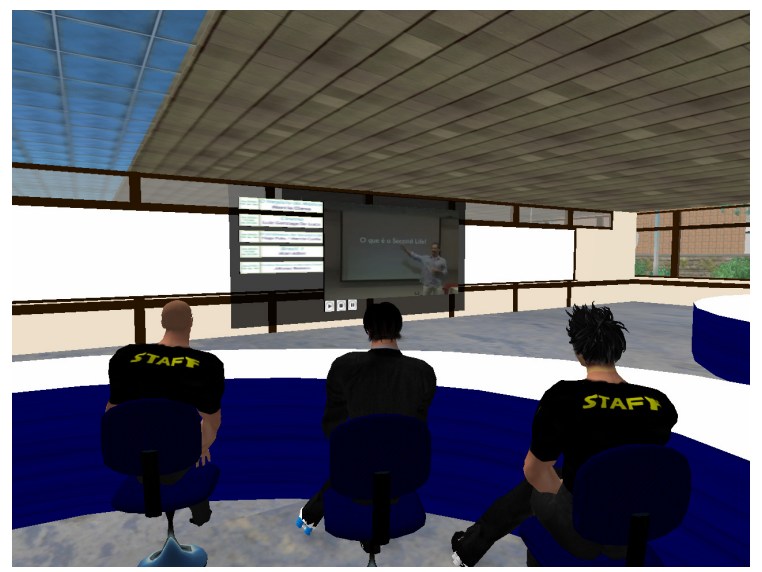

Figure 3 - Video on Demand for Avatars
Second Life [11] allows the real time interaction of its users by means of avatars in a space constructed and modified by the residents themselves. Besides acting as a platform of internal and external communication for companies and universities, Second Life makes it possible to launch prototypes and test user response before commercializing products in the real world.

The residents of Second Life are much more than simple users. They assume virtual identities, play roles, conduct virtual activities, participate in mass learning and collaboration and even create virtual businesses. It can be said that they are more than simple consumers, they are "prosumers" (consumers and producers) [17]. In Second Life consumers, besides co-innovating and co-producing the products they consume, also selforganize to create them. The most advanced users, in fact, no longer wait for an invitation to transform a product into a platform for their own innovations. They simply form their own online prosumer communities, share information, train those who are inexperienced, exchange information about products, collaborate in the creation of personalized products, trade them and exchange tips on how to modify them.

Educational institutions should follow the example of Linden Labs and of several large companies that have already moved into this environment to create an "academic product" that can motivate and qualify students to participate and add value to their academic subjects and projects in a massive scale. These opportunities to aggregate value must be extended to the entire curriculum of an individual's academic life.

\section{Computer Supported Collaborative Virtual Environments}

There is not a consensus regarding the definition of VR. Some authors try to list VR key elements, such as immersion (sensation of being in an environment), interactivity and sensorial feedback (visual, audible and tactile) [15]. Other authors describe VR as an advanced technique of user interface, where the user may navigate and interact with a computer generated 3D synthetic environment, being completely or partially immerse by the sensation generated by multi-sensorial channels [2].

Collaborative Virtual Environments are a special case of Virtual Reality Environments, where the emphasis is to provide distributed teams with a common virtual space where they can meet as if face-to-face, coexist and collaborate while sharing and manipulating, in real-time, the virtual artifacts of interest [9]. They can be seen as the result of a convergence of research interests within the Virtual Reality (VR) and CSCW communities.

Collaborative Virtual Environments has been studied for more than two decades [16] [1]. They have been used mainly for military training, academic research, and automotive and aircraft manufactures 
aiming to improve the overall product's quality and also aiming to reduce project's life cycle, cutting down costs and reducing the time-to-market of new products.

Only recently collaborative virtual environments left those restricted circles, becoming increasingly used through the Internet, especially due to the popularity of Second Life.

Google, Linden Lab, IBM, Cisco and many other large companies participate in the OpenID project [12] with the objective of creating a 3D WEB. Efforts are aimed at the establishment of open technical norms that will transform the Internet into a galaxy of independent, interconnected and linked virtual worlds through known protocols and APIs.

In this future scenario, it will be possible to go to a shopping mall to buy something with your friends at lunchtime even if you are miles away from it. In the real world you will be in a computer terminal at work, but the computer screen will take you to a digital replica of this mall. While you walk with your avatar through a virtual store, the store's web cameras will allow you to see its environment, sales clerks and store movement. Your Avatar will be configured with your real measurements, and when trying on, for example, a pair of jeans, you will be able to visualize if it suits you, and, if you want, to ask your accompanying friends for their opinions. The pair of jeans will be mailed to you.

The Institute Gartner [8] estimates that until 2011, $80 \%$ of Internet users and employees of large companies will have avatars, or digital replicas of themselves, to work, participate in communities and even play. Imagine what can be done for learning!

\section{Campus Online Project: Second Life Environment for the University Community}

The objective of this project is the re-creation in Second Life of the University Campus with a virtual company incubator, besides the construction of areas called SandBoxes for free creation by the students (Figure 4). The objective is to create an environment of innovation and exchange of knowledge and learning inside a virtual campus that will be an open participation platform where anything can be made and built. With Second Life we wish to create an environment where students produce $99 \%$ of the valuecreation of the subjects, courses and projects established in the Online Campus and even create their own virtual businesses.

Students will not be passive recipients as they are in classrooms; they will participate in the construction of content on an equal footing, creating value together with their collaborators and advisors. They will do this to satisfy their personal learning needs, to take part in communities that give them pleasure, to try to change the world or simply to have fun.

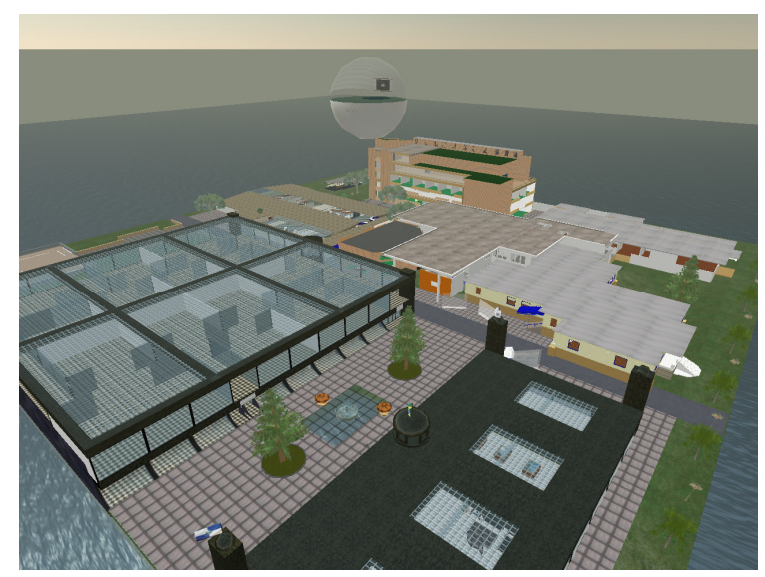

Figure 4 - Online Campus Aerial View

\subsection{Pilot Project}

To make possible the exchange of information in the Online Campus the construction of a critical mass of participants to attract more and more people to the environment is necessary [3]. In fact, few projects survive without constant financing and support or a central group of leaders to guide and manage the interactions. These actors supply the social capital and the technical infrastructure that other participants will develop. To extract long-term benefits from information-sharing communities such as Second Life it is necessary to create and always replenish the critical mass.

As a starting point, this project has built a virtual replica of the Business School (IAG - Portuguese acronym) that is characterized by the innovation aspect and by the understanding of how this unit present in the virtual world can relate to and complement the space created in Second Life. The objective of this environment is to investigate how this new form of relationship of the IAG with society can leverage business opportunities.

The pilot project currently under development has already acquired one private island in Second Life. On this island a virtual 3D environment with all the real buildings that represent IAG campus was developed. This representation is in a scale of 1 (one) to 2.5 (twopoint-five) to ensure a better perception of space in Second Life's environment, once an avatar has the ability to fly, take great leaps and cover vast distances quickly. All activities conducted inside Second Life will be reported externally to a Wiki [17] where all collaborators to this project will be able to share their experiences and record their work for future collaborators, thus helping to enhance the scientific and technological knowledge of the community to which they belong. 
Visitors and students will interact with the Online Campus as if in real life. For instance, a help person can be made available in the form of an avatar in a virtual reception desk to answer questions about the courses offered by the university and help register possible students in courses offered by the institution. In this reception desk, visitors have access to the entire content that is available today on the IAG website, including, for instance, course registration forms, in a different and innovative format (Figure 5).

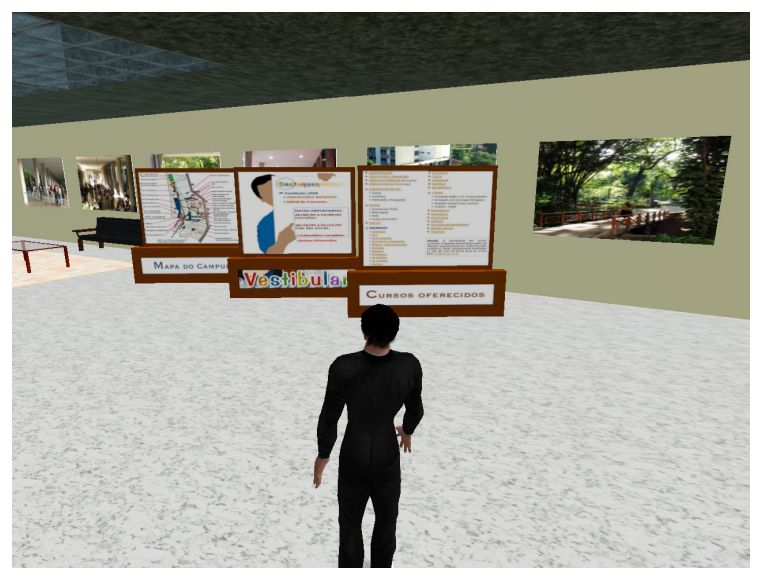

Figure 5 - Online Campus Information Billboards in the Reception Desk

This pilot project held the IAG's "Administration Week" event between September 25 and 27, 2007 in the Online Campus Floating Auditory (Figure 6). The students had their presence checked by the department's secretaries who were in the virtual world with their avatars. They asked questions to the presenters in the real world simultaneously to the event. All the event's lectures were recorded and are available in the Online Campus through streaming QuickTime video-ondemand. In those three days of the event the Online Campus had, on the average, over two thousand accesses a day.

Participants in future courses will have the advantage of simulating what they have learned in the classroom in a virtual world through the Virtual Company Incubator. From this Virtual Incubator people can interact with a company in the University Business incubator, contributing, therefore, for the transference to society of part of the results of this project. Simulations such as business-oriented games, stock exchange operations, risk environment simulations and case studies in diverse areas can be made available to students in Second Life, as well as opportunities to develop or work in the fastest-growing business areas in Second Life such as virtual marketing, telecommuting, job interviews and teleconferences (Figure 7).

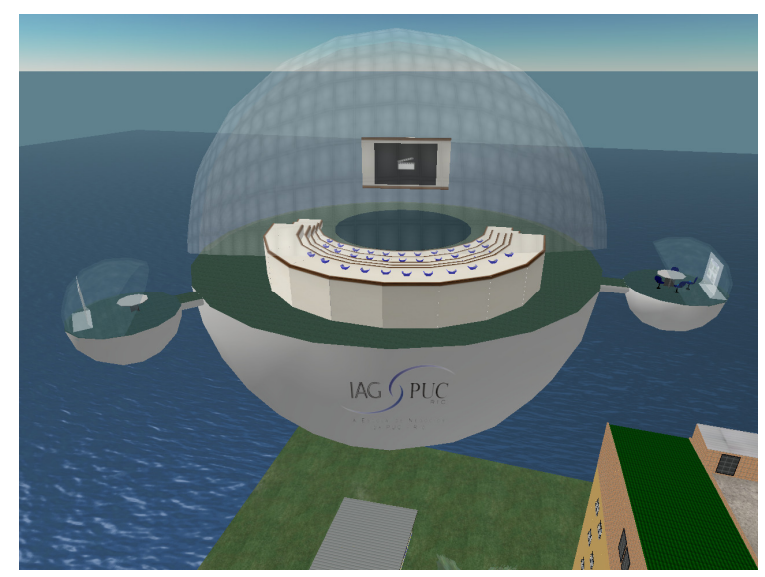

Figure 6 - Online Campus Floating Auditorium

\section{Conclusion}

The expected result is the opening of the university to new forms of cooperation, communication, coordination and construction of knowledge [5]. This opening is a new force for the university's growth and competitiveness. We hope that this virtual environment will stimulate students and researchers and increase the speed, reach and success of innovation, since in this virtual world everything is possible.

The idea is to create a global stage in which the local academic community and other universities' communities can create value, or even, who knows, new products in a highly synergic environment.

It is a part of this research project to establish the research and collaborative development models. It will be necessary within this stage of planning to reach an agreement about which departments and subjects will become candidates for a pioneering role.

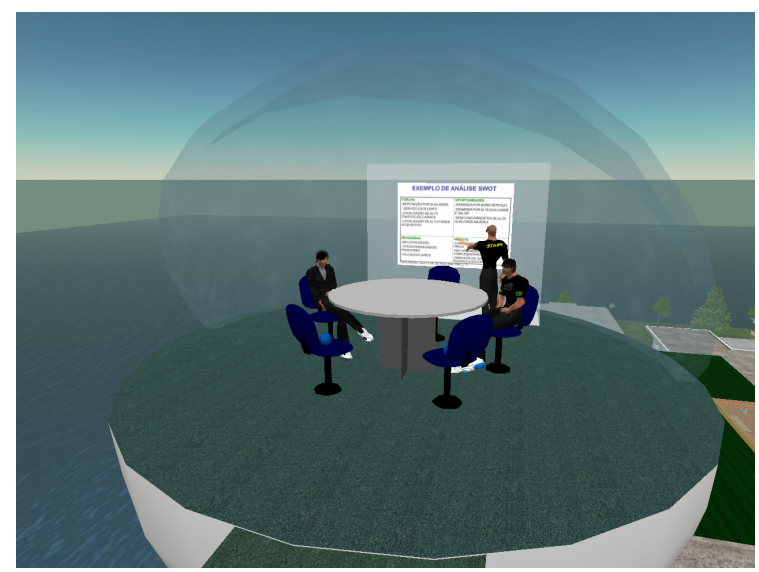

Figure 7 - Online Campus Floating Conference Room 
From the point-of view of educational and research programs at the university, there is a plan to include in Second Life subjects or activities from the Undergraduate and Graduate programs of the Department of Informatics. This measure will allow the informal continuation of the discussions initiated in class, making possible the contact among people in different spaces, and the accomplishment of e-learning actions in specific subjects.

\section{Acknowledgements}

This project is partially funded by FAPERJ PENSA Rio grant $\mathrm{n}^{\mathrm{o}} \mathrm{E}-26 / 110.339 / 2007$. It is also sponsored by Padre Leonel Franca Foundation and by the Brazilian Ministry of Science and Technology through the ESSMA project grant $n^{\circ} 552068 / 2002-0$. Hugo Fuks is sponsored by CNPq individual grant $\mathrm{n}^{\circ} 301917 / 2005-1$ and also receives grant from the FAPERJ project "Cientistas do Nosso Estado".

Tecgraf is a laboratory mainly funded by Petrobras.

\section{References}

[1] S. Benford, C. Greenhalgh, T. Rodden and J. Pycock. "Collaborative Virtual Environments", Communications of the ACM, 2001, 44(7): 79-85.

[2] G. Burdea and P. Coiffet, Virtual Reality Technology, John Wiley \& Sons, 1994.

[3] A. Dix. "Challenges for Cooperative Work on the Web: An Analytical Approach", Computer Supported Cooperative Work (CSCW): The Journal of Collaborative Computing, 1997, 6(2-3): 135-156.

[4] H. Fuks, M. A. Gerosa, C. G. Barreto and C. J. P. Lucena, "Middleware de Integração entre o Ambiente AulaNet e o Ginga", SET 2007 - Congresso da Sociedade Brasileira de Engenharia de Televisão, São Paulo, 2007.

[5] H. Fuks, A. Raposo, M. A. Gerosa and C. J. P. Lucena, "Applying the 3C Model to Groupware Development", International Journal of Cooperative Information Systems (IJCIS), 2005, 14(2-3): 299-328, World Scientific.

[6] D. Filippo, A. Raposo, M. Endler and H. Fuks, "Ambientes Colaborativos de Realidade Virtual e Aumentada", in: Realidade Virtual e Aumentada Conceitos, Projeto e Aplicações, Cláudio Kirner and Robson Siscoutto (eds), Editora SBC - Sociedade Brasileira de Computação, 2007, Chap. 9, pp 169-192.

[7] D. Filippo, C. G. Barreto, H. Fuks and C. J. P. Lucena, "Collaboration in Learning with Mobile Devices: Tools for Forum Coordination", 22nd ICDE - World Conference on Distance Education: Promoting Quality in On-line, Flexible and Distance Education (CD-ROM), Publisher: ABED, Rio de Janeiro, Brazil, 2006

[8] Gartner, 2007, http://www.gartner.com/, Visited at $23 / 10 / 2007$

[9] G. Goebbels, V. Lalioti and M. Göbel. "Design and Evaluation of Team Work in Distributed Collaborative Virtual Environments", Proceedings of the ACM
Symposium on VRST'03, Osaka, Japan, 2003, pp. 231238.

[10] Harvard, 2006, http://slurl.com/secondlife/Berkman/114/53/24, Visited at 23/10/2007

[11] LINDEN, 2007, http://www.secondlife.com, Visited at $23 / 10 / 2007$

[12] OpenID, 2007, http://www.openid.org, Visited at 23/10/2007

[13] M. Pimentel, H. Fuks and C. J. P. Lucena, "Mediated Chat Development Process: Avoiding Chat Confusion on Educational Debates", Computer Supported Collaborative Learning, Taiwan, Lawrence Erlbaum Associates, pp. 499-503, July, 2005

[14] M. Rymaszewski, W. J. Au, C. Ondrejka and R. Platel, Second Life: The Official Guide, Publisher Wiley, 2007

[15] W. R. Sherman, and a. B. Craig, Understanding Virtual Reality: Interface, Application, and Design, Morgan Kaufmann, 2003.

[16] S. K. Singhal and M. Zyda. Networked Virtual Environments: Design and Implementation. New York: ACM Press, 1999

[17] D. Tapscott, Wikinomics: how mass collaboration changes everything, Publisher Potfolio, 2007 